\title{
Spectroscopic factors and asymptotic normalization coefficients in mirror three-body systems
}

\author{
N. K. Timofeyuk ${ }^{1}$ and I. J. Thompson ${ }^{2}$ \\ ${ }^{1}$ Physics Department, University of Surrey, Guildford, Surrey GU2 7XH, England, United Kingdom \\ ${ }^{2}$ Lawrence Livermore National Laboratory, P. O. Box 808, L-414, Livermore, California 94551, USA
}

(Received 12 June 2008; published 26 November 2008)

\begin{abstract}
Using a three-body model, we study the dependence of spectroscopic factors for the overlap integrals $\langle$ core $+N|$ core $+N+N\rangle$ on the binding energy of the core $+N$ subsystem, considering as prototypes ${ }^{6} \mathrm{He},{ }^{6} \mathrm{Be},{ }^{9} \mathrm{Li},{ }^{9} \mathrm{C},{ }^{18} \mathrm{O}$, and ${ }^{18} \mathrm{Ne}$. We show that at small $N$-core binding energies these spectroscopic factors can be strongly influenced by the geometrical mismatch between the two-body $N$-core wave function that stretches into the classically forbidden region and the spatially confined three-body function. This mismatch comes from the strong two-body correlations between the nucleons outside the core and due to the core recoil effects. The mismatch leads to symmetry breaking in mirror spectroscopic factors that in some cases can be large enough to be observed in nucleon removal reactions. It is also responsible for deviations of the ratios of mirror asymptotic normalization coefficients (ANCs) from the simple model-independent analytical estimates. We discuss the influence of such mirror symmetry breaking on the prediction of direct stellar $(p, \gamma)$ reactions from the measured mirror neutron ANCs.
\end{abstract}

DOI: 10.1103/PhysRevC.78.054322

PACS number(s): 21.10.Jx, 21.45.-v, 27.20.+n

\section{INTRODUCTION}

It has been considered for a long time that mirror nuclear states have very similar properties. In particular, it is widely assumed that spectroscopic factors for mirror states are the same. This assumption is often exploited for various purposes including prediction of nuclear reactions in stars, such as proton capture, that cannot be directly measured.

To predict the rates for nonresonant peripheral capture reactions, the knowledge of spectroscopic factors alone is not sufficient as their cross sections are determined by asymptotic normalization coefficients (ANCs) [1]. It has been recently shown that ANCs in mirror nuclear states can be related by an approximate model-independent analytical expression that involves only mirror nucleon separation energies, the nuclear charge, and the radius of the interaction between the last nucleon and residual nucleus [2]. This link gives possibility to predict the $(p, \gamma)$ cross sections using mirror neutron ANCs measured in peripheral neutron transfer reactions and it has already been used in Refs. [3-5].

Recently, a study performed within the Gamow shell model [6] has shown that coupling to the continuum leads to a specific behavior of spectroscopic factors that, when plotted against the one-nucleon separation energy, resembles the cusps seen in scattering and reaction cross sections in the vicinity of a reaction threshold. According to Ref. [6], spectroscopic factors for one-nucleon overlaps involving near-threshold states can significantly differ from shell-model predictions. Consequently, the spectroscopic factors in mirror states, one of which has strongly bound neutrons and the other loosely bound protons, may not be equal anymore because, in the first case, no significant deviations from the standard shell model are expected, whereas in the second one such deviations may occur.

The symmetry breaking in spectroscopic factors, that may occur in mirror pairs involving threshold states, can lead to deviations between the squared ratio of their ANCs, $\left(C_{p} / C_{n}\right)^{2}$, where $C_{p}\left(C_{n}\right)$ is the proton (neutron) $\mathrm{ANC}$, and the analytical expression

$$
\mathcal{R}_{0}=\left|\frac{F_{l}\left(i \kappa_{p} R_{N}\right)}{\kappa_{p} R_{N} j_{l}\left(i \kappa_{n} R_{N}\right)}\right|^{2}
$$

derived in Ref. [2] as an approximation for $\left(C_{p} / C_{n}\right)^{2}$. In this expression, $\kappa_{p}\left(\kappa_{n}\right)$ is the wave number for removed proton (neutron), $F_{l}$ is the regular Coulomb function, $j_{l}$ is the spherical Bessel function for the orbital momentum $l$ and $R_{N}=1.3 B^{1 / 3}$, where $B$ is the mass of the remainder nucleus. Equation (1) has been obtained in Ref. [2] assuming equality of internal wave functions in mirror decay channels, which normally occurs if mirror spectroscopic factors are equal. Therefore, determinations of the stellar $(p, \gamma)$ cross sections using Eq. (1) can be less accurate if mirror spectroscopic factors differ significantly.

One class of nuclei where the symmetry-breaking effects in mirror spectroscopic factors would be of particular interest is the mirror pairs in which the proton rich partners have one-proton decay threshold just under the two-proton decay threshold, for example, as in ${ }^{9} \mathrm{C}$ or in ${ }^{18} \mathrm{Ne}$. In such nuclei, the nuclear structure is driven by explicit two-proton dynamics and the core $+p+p$ configuration becomes very important. Previously, we have shown that near the core $+N$ threshold the overlap integral $\langle$ core $+N|$ core $+N+N\rangle$ can converge to the correct asymptotic behavior slower than in normal nuclei $[7,8]$. This can directly affect the interpretation of one-nucleon removal reactions as well as predictions for $(p, \gamma)$ direct capture on weakly bound nuclei. In particular, at astrophysical energies, the $(p, \gamma)$ reactions occur at the region where a true asymptotic behavior of overlap integrals is achieved and their cross sections are determined by normal ANCs. These ANCs are determined experimentally from transfer, breakup or Coulomb dissociation reactions. Although such reactions are often peripheral, they are also sensitive to the near-surface part of the overlap integral that can be influenced 
by preasymptotic abnormalities. If the latter are significant, then what is extracted from such reactions may not correspond to the true $\mathrm{ANCs}$. In these cases, determining $C_{p}$ from a mirror neutron ANC can be very helpful because the mirror system core $+n+n$ is always more bound than the core $+p+p$ and possible threshold effects leading to nonstandard convergence of the strongly bound neutron overlaps should be absent. Examples are the stellar ${ }^{8} \mathrm{~B}(p, \gamma){ }^{9} \mathrm{C}$ and the ${ }^{17} \mathrm{~F}(p, \gamma){ }^{18} \mathrm{Ne}$ reactions. The ${ }^{9} \mathrm{C}$ and ${ }^{18} \mathrm{Ne}$ ANCs can be determined using ANCs for ${ }^{8} \mathrm{Li}$ and ${ }^{18} \mathrm{O}$ (in fact, the ${ }^{9} \mathrm{C}$ ANC has been already determined in this way in Ref. [3]). Therefore, it is important to study the accuracy of the approximation $\left(C_{p} / C_{n}\right)^{2} \approx \mathcal{R}_{0}$ and its correlations with possible symmetry breaking in mirror spectroscopic factors for such systems.

In this article, we study mirror spectroscopic factors and mirror ANCs within a three-body model where exact methods to solve the Schrödinger equation exist. Three-body models are widely used when describing dynamics of systems in which three-body degrees of freedom are important, for example, in breakup of Borromean nuclei, in two-proton radioactivity, in treatment of the continuum effects within the continuum-discretized coupled-channels approach, etc. Although these models miss some other aspects of nuclear structure, such as mixture of nonorthogonal configurations, their contribution to explaining observables influenced by the three-body dynamincs is very valuable. In Sec. II we briefly present the three-body model and the interactions used. In Sec. III, the asymptotic convergence of overlap integrals is discussed. In Sec. IV, by artificially changing the strength of the core- $N$ interaction we study energy dependence and threshold effects in the spectroscopic factors. Section V studies the ratio $\mathcal{R}_{0}$ for mirror systems core $+p+p$ and core $+n+n$ and the results obtained are discussed in Sec. VI.

\section{THREE-BODY MODEL}

We have chosen as prototypes the following mirror pairs:

(i) ${ }^{6} \mathbf{H e}-{ }^{6} \mathbf{B e}$. The energy behavior of the ${ }^{6} \mathrm{He}$ spectroscopic factor was studied in the Gamow shell model in Ref. [6]. We check whether the three-body model predicts the same general trend in spectroscopic factors near the ${ }^{5}$ He threshold.

(ii) ${ }^{9} \mathbf{L i}-{ }^{9} \mathbf{C}$. The ${ }^{9} \mathrm{C}$ ANC determines the rate of the ${ }^{8} \mathrm{~B}(p, \gamma){ }^{9} \mathrm{C}$ reaction that is a part of the hot $p p$ chain in the explosive hydrogen burning. This ANC was recently calculated in Ref. [3] using the approximation (1) and the ${ }^{9} \mathrm{Li}$ ANC, determined from analysis of the ${ }^{8} \mathrm{Li}(d, p){ }^{9} \mathrm{Li}$ transfer reaction.

(iii) ${ }^{18} \mathbf{O}-{ }^{18} \mathrm{Ne}$. These nuclei are traditionally considered as classical shell-model nuclei with two valence nucleons in the $s d$ shell. However, three-body dynamics should be important in ${ }^{18} \mathrm{Ne}$ because its ${ }^{16} \mathrm{O}+p$ subsystem is weakly bound. We study two $0^{+}$, two $2^{+}$, and one $4^{+}$low-lying states for this mirror pair. The $2^{+}$ states, formed in the stellar reaction ${ }^{17} \mathrm{~F}(p, \gamma){ }^{18} \mathrm{Ne}$, are important for understanding the nucleosynthesis in novae.

\section{A. Model}

We performed three-body calculations for ${ }^{4} \mathrm{He}+n+n$, ${ }^{4} \mathrm{He}+p+p,{ }^{7} \mathrm{Li}+n+n,{ }^{7} \mathrm{Be}+p+p,{ }^{16} \mathrm{O}+n+n$, and ${ }^{16} \mathrm{O}+p+p$ systems assuming no internal structure for the ${ }^{4} \mathrm{He},{ }^{7} \mathrm{Li},{ }^{7} \mathrm{Be}$, and ${ }^{16} \mathrm{O}$ cores but taking antisymmetrization into account by eliminating forbidden core- $N$ states with the Pauli projection technique. The three-body Schrödinger equation,

$$
\begin{aligned}
\left(T_{n n}\right. & +T_{\text {core-nn }}+V_{\text {core-n }}\left(\left|\boldsymbol{r}_{c}-\boldsymbol{r}_{N_{1}}\right|\right)+V_{\text {core }-n}\left(\left|\boldsymbol{r}_{c}-\boldsymbol{r}_{N_{2}}\right|\right) \\
& \left.+V_{n n}\left(\left|\boldsymbol{r}_{N_{1}}-\boldsymbol{r}_{N_{2}}\right|\right)-E\right) \Psi_{3}=0,
\end{aligned}
$$

has been solved by expanding the wave function $\Psi_{3}$ onto the hyperspherical harmonics $(\mathrm{HH})$ basis [9],

$$
\Psi_{3}=\rho^{-5 / 2} \sum_{K l_{x} l_{y} \mathrm{LS}} \chi_{K l_{x} l_{y}}^{\mathrm{LST}}(\rho) \mathcal{Y}_{K l_{x} l_{y} \operatorname{LSTJM}}\left(\Omega_{5}\right),
$$

where $\mathcal{Y}_{K l_{x} l_{y} \mathrm{LSTJM}}\left(\Omega_{5}\right)$ are the hyperspherical harmonics combined with spin and isospin wave function; $\rho$ is the hyperradius; $\Omega_{5}$ is a set of hyperangles; $K$ is hypermoment; $l_{x}\left(l_{y}\right)$ is the orbital momentum of the core- $N[($ core $+N)-N]$ system; $L, S, T$, and $J$ are the total orbital momentum, spin, isospin, and total angular momentum, respectively; and $M$ is the projection of the total angular momentum. Usually, the hyperradial part $\chi_{K \gamma}(\rho)$ is found by solving the coupled set of differential equations

$$
\begin{array}{r}
\left\{-\frac{\hbar^{2}}{2 m}\left[\frac{d^{2}}{d \rho^{2}}-\frac{\mathcal{L}_{K}\left(\mathcal{L}_{K}+1\right)}{\rho^{2}}\right]-E\right\} \chi_{K \gamma}(\rho) \\
=\sum_{K^{\prime} \gamma^{\prime}} V_{K \gamma, K^{\prime} \gamma^{\prime}}(\rho) \chi_{K^{\prime} \gamma^{\prime}}(\rho),
\end{array}
$$

where $\gamma \equiv\left\{l_{x} l_{y} L S T\right\}, V_{K \gamma, K^{\prime} \gamma^{\prime}}(\rho) \equiv\left\langle\mathcal{Y}_{K \gamma}\left(\Omega_{5}\right)|V| \mathcal{Y}_{K^{\prime} \gamma^{\prime}}\left(\Omega_{5}\right)\right\rangle$ is the hyperradial potential and $\mathcal{L}_{K}=K+3 / 2$. However, when bound eigenstates of the core- $N$ potential are present is forbidden partial waves, the Schrödinger equation should be solved in a subspace of allowed states. Details of the techique that deals with this problem are given in Ref. [10].

Ideally, the summation over $K$ in expansion (3) should go up to infinity. In reality, this sum has to be truncated up to some $K_{\max }$. In the present article, the model space has been truncated to $K_{\max }=50$ in most cases but reduced to $K_{\max }=26$ and $K_{\max }=24$ for ${ }^{18} \mathrm{O}\left(2^{+}\right)-{ }^{18} \mathrm{Ne}\left(2^{+}\right)$and ${ }^{18} \mathrm{O}\left(4^{+}\right)-{ }^{18} \mathrm{Ne}\left(4^{+}\right)$, respectively.

\section{B. Choice of potentials}

For the test case of ${ }^{6} \mathrm{He}-{ }^{6} \mathrm{Be}$, the $V_{n n}$ potential was represented by just one Gaussian that gives a scattering length of $-20 \mathrm{fm}$. For all other nuclei, the GPT potential [11], that includes central, spin-orbit, and tensor interactions, was used in $l \leqslant 2$ partial waves.

For the $V_{\text {core }-n}$ interactions, we used the Woods-Saxon potentials with the standard set of radius $\left(r_{0}=1.25 \mathrm{fm}\right)$ and diffuseness $(a=0.65 \mathrm{fm})$. The depths of the central potentials for allowed states have been artificially varied to get different separation energies in the core $+N$ systems. The spin-orbit potential for the $0 d$ state in ${ }^{18} \mathrm{O}_{-}{ }^{18} \mathrm{Ne}$ has a fixed depth of $5.5 \mathrm{MeV}$ and the same $r_{0}$ and $a$ as the central potentials. No 
spin-orbit potential was used for ${ }^{6} \mathrm{He}^{6}{ }^{6} \mathrm{Be}$ and ${ }^{9} \mathrm{Li}-{ }^{9} \mathrm{C}$, to make all components bound. The depth of the central Woods-Saxon potential for forbidden $0 s$ state was $-70 \mathrm{MeV}$ and $-60 \mathrm{MeV}$ for ${ }^{6} \mathrm{He}-{ }^{6} \mathrm{Be}$ and ${ }^{9} \mathrm{Li}-{ }^{9} \mathrm{C}$, respectively, and $-56.55 \mathrm{MeV}$ for forbidden $0 p$ states in ${ }^{18} \mathrm{O}-{ }^{18} \mathrm{Ne}$. A spin-orbit potential with the depth of $8.5 \mathrm{MeV}$ has been included for the latter. Such a choice provides correct single-particle energies of the forbidden states (except for the $0 s$ state in ${ }^{18} \mathrm{O}-{ }^{18} \mathrm{Ne}$ at the ${ }^{17} \mathrm{~F}$ binding energies different from experimental ones). For ${ }^{6} \mathrm{Be},{ }^{9} \mathrm{C}$, and ${ }^{18} \mathrm{Ne}$, the Coulomb $p$-core and $p p$ potentials have been also included.

\section{OVERLAP INTEGRALS AND THEIR ASYMPTOTIC CONVERGENCE}

The overlap integral $\langle$ core $+N|$ core $+N+N\rangle$ is obtained by overlapping the two-body and three-body wave functions,

$$
I(\boldsymbol{r})=\left\langle\psi_{2} \mid \Psi_{3}\right\rangle
$$

and the spectroscopic factor is determined then as

$$
S_{l j}=\int_{0}^{\infty} d r r^{2} I_{l j}^{2}(r),
$$

where $I_{l j}(r)$ is the radial part of $I(\boldsymbol{r})$. Asymptotically, this overlap should behave as

$$
I_{l j}(r) \approx C_{l j} \frac{W_{-\eta, l+1 / 2}(2 \kappa r)}{r}, \quad r \rightarrow \infty,
$$

where $C_{l j}$ is the ANC, $W$ is the Whittaker function, $\kappa=$ $\left(2 \mu \epsilon / \hbar^{2}\right)^{1 / 2}, \epsilon$ is the difference in binding energy between nuclei core $+N+N$ and core $+N, \mu$ is the reduced mass for the $($ core $+N)+N$ system, $\eta=Z_{\text {core }+N} Z_{N} e^{2} \mu / \hbar^{2} \kappa, Z_{i}$ is the charge of the nucleus $i$ and $j$ is the total relative angular momentum between the core $+N$ system and the removed nucleon.

The question of whether the asymptotic behavior given by (7) can be achieved by the HH expansion of the three-body wave function deserves a special discussion. Formally, the $\mathrm{HH}$ basis is complete on the hypersphere of fixed hyperradius. So at any given value of $\rho$ one can reproduce the wave function with any precision by increasing the number of basis HHs [12]. Using the HH expansion from Ref. [9] it is easy to show that the overlap $I_{l j}(r)$ is represented by a sum of the following integrals

$$
\int_{0}^{\infty} d \rho_{c} \psi_{2}\left(\rho_{c}\right) \chi_{K l_{x} l_{y}}^{\mathrm{LST}}\left(\sqrt{\rho_{c}^{2}+\frac{m_{c+1}}{m_{c+2}} r^{2}}\right) f_{K l_{x} l_{y}}\left(\rho_{c}, r\right),
$$

where $\rho_{c}=\sqrt{\frac{m_{c}}{m_{c+1}}} r_{\text {core }-N}, \rho^{2}=\rho_{c}^{2}+\frac{m_{c+1}}{m_{c+2}} r^{2}, m_{i}$ is the mass number of the particle $i$ and $f_{K l_{x} l_{y}}\left(\rho_{c}, r\right)$ is a geometrical function that contains only some powers of $\rho_{c} / \rho$ and $r / \rho$ and the Jacobi polynomials. At large $r$, all the physical information about the overlap $I_{l j}(r)$ comes from $\rho_{c}$ 's that maximize the product $\psi_{2}\left(\rho_{c}\right) \chi_{K l_{x} l_{y}}^{\mathrm{LST}}\left(\sqrt{\rho_{c}^{2}+\frac{m_{c+1}}{m_{c+2}} r^{2}}\right)$. This occurs at $r_{\text {core }-N}$ that are within the range of the $N$-core interaction. Therefore, the asymptotic hehavior for $I_{l j}(r)$ is linked to the $\chi_{K l_{x} l_{y}}^{\mathrm{LST}}$ behavior at large $\rho$.

It is well-known that in the absence of the Coulomb interactions the asymptotics of $\Psi_{3}$ for three separated particles is $\exp (-k \rho) / \rho^{5 / 2}$, where $k$ is determined by the three-body energy. This may give an impression that the asymptotics (7) in the binary channel can never be reached in the HH expansion of $\Psi_{3}$. However, the hyperradial functions $\chi_{K l_{x} l_{y}}^{\mathrm{LST}}(\rho)$ will achieve such a behavior only at $\rho$ for which $\left|V_{K \gamma, K^{\prime} \gamma^{\prime}}(\rho)\right| \ll|E|$ and $\frac{\hbar^{2}}{2 m} \frac{\mathcal{L}_{K}\left(\mathcal{L}_{K}+1\right)}{\rho^{2}} \ll|E|$. In general, such a value for $\rho$ does not exist. There are two reasons for this.

(i) At large $\rho$, two of three particles can still be next to each other and strongly interact, which leads to slow decrease of $V_{K \gamma, K^{\prime} \gamma^{\prime}}(\rho)$. Their dependence on $\rho$ and $K$ can be easily understood for Gaussian potentials of the range $\alpha$, where the most important hyperradial potentials are $V_{K \gamma, K^{\prime} \gamma^{\prime}}(\rho) \sim \frac{\sqrt{\Gamma(K+3 / 2) \Gamma(K+3 / 2)}}{\Gamma\left(\left(K+K^{\prime}+3\right) / 2\right)}{ }_{1} F_{1}\left(\frac{3}{2}, \frac{K+K^{\prime}+3}{2},-\frac{2 \rho^{2}}{\alpha^{2}}\right)$. At large $\rho, V_{K \gamma, K^{\prime} \gamma^{\prime}}(\rho) \sim 1 / \rho^{3}$. The strength of the diagonal terms increases as $K^{3 / 2}$ at a fixed large $\rho$ and a similar increase occurs for nondiagonal terms that link two neighboring $K$ and $K^{\prime}$. As the result, for any $\rho$ one can find $K$ where some hyperradial potentials would be comparable to $E$. The situation is even more complicated because the $1 / \rho^{5}, 1 / \rho^{7}$ and other terms in $V_{K \gamma, K^{\prime} \gamma^{\prime}}(\rho)$ grow with large $K$.

(ii) For any arbitrarily fixed $\rho$ one can find $K$ such that $\frac{\hbar^{2}}{2 m} \frac{\mathcal{L}_{K}\left(\mathcal{L}_{K}+1\right)}{\rho^{2}}=|E|$ so that the centrifugal barrier will dominate over the three-body energy and the hyperradial functions corresponding to larger $K$ 's will not behave as $\exp (-k \rho) / \rho^{5 / 2}$. As the result, the $\chi_{K l_{x} l_{y}}^{\mathrm{LST}}(\rho)$ behavior at large $\rho$ will depend on the interplay between (i) and (ii).

The present calculations have been performed for $\rho_{\max }=$ $30 \mathrm{fm}$. The analysis of $V_{K \gamma, K^{\prime} \gamma^{\prime}}(\rho)$ for a few arbitrary chosen cases has confirmed that at $\rho_{\max }=30 \mathrm{fm}$ and $K_{\max }=50$ the diagonal hyperradial potentials can be as large as $16 \%$ of the three-body energy and the nondiagonal potentials can comprise up to one third of it. Also, the centrifugal barriers for large $K$ are significantly larger than $E$. As the result, the calculated functions $\chi_{K l_{x} l_{y}}^{\mathrm{LST}}(\rho)$ do not expose the asymptotic three-body behavior $\exp (-k \rho) / \rho^{5 / 2}$ but are governed by the three-body dynamics. It is this deviation from $\exp (-k \rho) /$ $\rho^{5 / 2}$ that participate in formation of the binary channel. Therefore, to calculate the overlap $I_{l j}(r)$ at large $r$, a sufficiently large number of $\chi_{K l_{x} l_{y}}^{\mathrm{LST}}(\rho)$ should be taken into account to provide the precision required for a given application. It can happen for some physical cases that the computational power may expire faster than such a precision is achieved. In this case, extended $\mathrm{HH}$ expansion with the binary channel has been formulated in Ref. [12], which allows to achieve the convergence for $I_{l j}(r)$ much faster. However, for the purposes of the present study, we find that convergence of $I_{l j}(r)$ obtained by a standard $\mathrm{HH}$ expansion is sufficient to make the physical conclusions that follow.

The typical convergence of the ratio $C_{l j}(r)$ of the overlap integrals $I_{l j}(r)$ to its asymptotic form given by Eq. (7) is shown in Fig. 1 for the case of $\left\langle\left.{ }^{5} \mathrm{Li}\right|^{6} \mathrm{Be}\right\rangle$. The calculations presented in this figure have been performed with the $p$ - ${ }^{4} \mathrm{He}$ interaction that binds ${ }^{5} \mathrm{Li}$ by $35 \mathrm{keV}$. To get the converged result up to $r=25 \mathrm{fm}$, we have to use $K_{\max }=50$. The numerical accuracy of the ANC for $K_{\max }=50$ is this case is about $0.02 \%$. In other calculations using the same model space, the accuracy is $\sim 0.1 \%$ for ${ }^{9} \mathrm{Li}$ and ${ }^{9} \mathrm{C}$, and $\sim 0.2 \%$ for ${ }^{18} \mathrm{O}\left(0^{+}\right)$and ${ }^{18} \mathrm{Ne}\left(0^{+}\right)$. 


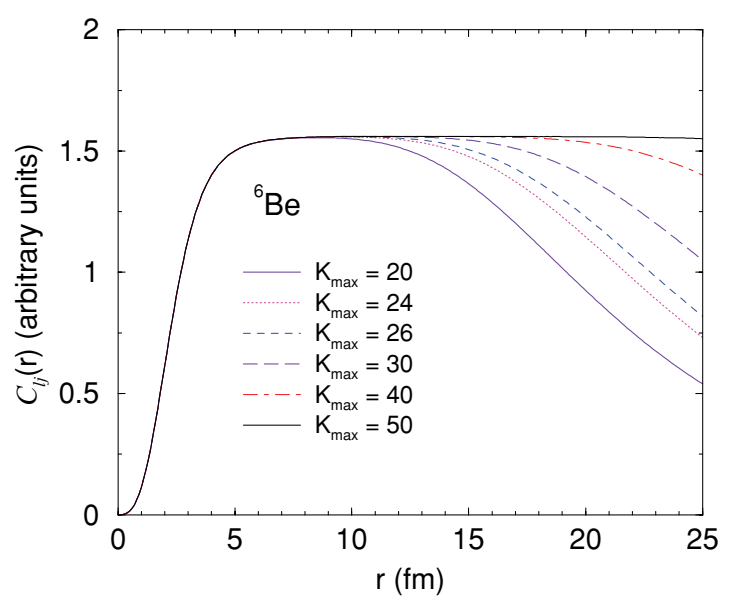

FIG. 1. (Color online) Ratio $C_{l j}(r)=I_{l j}(r) /\left(W_{-\eta, l+1 / 2}(2 \kappa r) / r\right)$ for the $\left\langle{ }^{5} \mathrm{Li} \mid{ }^{6} \mathrm{Be}\right\rangle$ overlap for different model spaces $K_{\max }$. The binding energy of ${ }^{5} \mathrm{Li}$ is $0.035 \mathrm{MeV}$.

However, in many physical applications such a precision is not required so that $K_{\max }$ could be reduced. This is useful for the cases where the number of $\mathrm{HH}$ channels is too large. For example, for $2^{+}$and $4^{+}$states in ${ }^{18} \mathrm{O}$ and ${ }^{18} \mathrm{Ne}$, where $K_{\max }$ is only 26 and 24 , the estimated uncertainty of the ANCs, determined here as maximum value of $C_{l j}(r)$, is about $0.5 \%$, which is still accurate enough for physical applications. And, finally, we have to note that the binding energies and the spectroscopic factors converge much earlier, by $K_{\max }=20$, because these quantities do not depend very strongly on the wave function behavior at large distances.

\section{ENERGY DEPENDENCE OF SPECTROSCOPIC FACTORS}

In this work, only the overlap integrals between the bound states of three- and two-body states are considered, as the overlaps involving continuum states deserve a separate publication. The calculated spectroscopic factors for one-neutron overlaps (no Coulomb interaction) are shown in Fig. 2 as function of bound state energies in the core $+n$ subsystem.

\section{A. ${ }^{6} \mathrm{He}$}

We artificially bind ${ }^{5} \mathrm{He}$ in our study and neglect the spinorbit interaction to avoid the problem of the ${ }^{5} \mathrm{He}\left(\frac{1}{2}^{-}\right)$spectroscopic factor in continuum for small separation energies in ${ }^{5} \mathrm{He}\left(\frac{3}{2}^{-}\right)$. Therefore, we show the sum of the spectroscopic factors, $S_{\frac{3}{2}}+S_{\frac{1}{2}}$. The strong near-threshold decrease of the spectroscopic factor, similar to that obtained in Ref. [6] within the Gamow shell model, is clearly seen in Fig. 2(a) (circles). We have done several other calculations which demonstrate that the origins of this decrease are the $n n$-correlations and the ${ }^{4} \mathrm{He}$-core recoil effect. When we set the ${ }^{4} \mathrm{He}$ mass to a very large number and assume $V_{n n}=0$, then energy dependence of the spectroscopic factor disappears [squares in Fig. 2(a)] because such a case corresponds to a standard mean-field calculation where the core $+n+n$ wave function is just a product of two single-particle wave functions and the total energy is the sum of two single-particle energies. The energy dependence and the threshold effect reappear when either the original mass of ${ }^{4} \mathrm{He}$ (triangles down) or the $V_{n n}$ interaction (triangles up) are restored.

The energy dependence of spectroscopic factor obtained in exact calculations for infinitely heavy core can be reproduced in a simple model in which the three-body wave function $\Psi_{3}$ is replaced by a Hartree-Fock type wave function $\Psi_{\mathrm{HF}}=$ $\mathcal{A}\left[\varphi_{\text {eff }}\left(\boldsymbol{r}_{1}\right) \varphi_{\text {eff }}\left(\boldsymbol{r}_{2}\right)\right]$, where $\varphi_{\text {eff }}(\boldsymbol{r})$ is an effective single-particle $0 p$ wave function belonging to a mean-field Hamiltonian that accounts for increase of binding due to the strong $N N$ correlations. The single-particle Hartree-Fock energies are equal to $E\left({ }^{6} \mathrm{He}\right)-E\left({ }^{5} \mathrm{He}\right)$ thus providing a correct asymptotics for the $\left\langle{ }^{5} \mathrm{He} \mid{ }^{6} \mathrm{He}\right\rangle$ overlap. The spectroscopic factor of such a model is equal to the square of the overlap between the radial parts of $\varphi_{\text {eff }}(\boldsymbol{r})$ and the two-body wave function $\psi_{2}(\boldsymbol{r})$ of ${ }^{5} \mathrm{He}$ :

$$
S \approx S_{\mathrm{eff}} \equiv\left[\int_{0}^{\infty} d r r^{2} \varphi_{\mathrm{eff}}(r) \psi_{2}(r)\right]^{2} .
$$

We generate $\varphi_{\text {eff }}(r)$ using the Woods-Saxon potentials with same geometry that has been used to calculate $\psi_{2}(r)$ but increase their depths to reproduce the $E\left({ }^{6} \mathrm{He}\right)-E\left({ }^{5} \mathrm{He}\right)$ values obtained in three-body calculations. The resulting effective spectroscopic factor $S_{\text {eff }}$, shown in Fig. 3, differs from the one obtained in exact three-body calculations by no more than $2 \%$. For finite mass core, it is more difficult to construct a proper effective model that would account for the core recoil correctly. Nevertheless, we have managed to explain the energy dependence of the spectroscopic factor in this case by assuming that the wave functions $\varphi_{\mathrm{eff}}(r)$ and $\psi_{2}(r)$ correspond to different reduced masses, $5 / 6$ and $4 / 5$, respectively, and that the potential well for $\varphi_{\text {eff }}(r)$ has larger radius, $r_{0}=1.3 \mathrm{fm}$ and diffuseness, $a=0.75 \mathrm{fm}$. However, the resulting effective spectroscopic factor should be reduced by a factor of 0.9 . This factor may appear due to the weight of $\Psi_{\mathrm{HF}}$ in the exact wave function $\Psi_{3}$.

\section{B. ${ }^{9} \mathbf{L i}$}

The ${ }^{7} \mathrm{Li}$ core has nonzero spin, is deformed, and can be easily excited to the nearest state $\frac{1}{2}^{-}$. For nonzero spin of the core, the hyperspherical harmonics expansion contains too many channels so that it becomes impossible to perform converged calculations. Therefore, we have neglected the ${ }^{7} \mathrm{Li}$ spin and assumed that ${ }^{7} \mathrm{Li}$ is spherical. As in the ${ }^{6} \mathrm{He}$ case, we neglect the $n{ }^{7} \mathrm{Li}$ spin-orbit interaction to avoid the problem of the ${ }^{8} \mathrm{Li}$ spectroscopic factor in continuum for small $n-{ }^{7} \mathrm{Li}$ binding energies. The resulting spectroscopic factor, defined as a sum $S_{\frac{3}{2}}+S_{\frac{1}{2}}$ is shown in Fig. 2(b) by circles as a function of bound state energy in ${ }^{8} \mathrm{Li}$. This spectroscopic factor is larger than that of ${ }^{6} \mathrm{He}$ because recoil effects are weaker; however, it still exhibits near-threshold decrease as in the ${ }^{6} \mathrm{He}$ case. Neglecting the ${ }^{7} \mathrm{Li}$ spin and the core excitations leads to overbound ${ }^{9} \mathrm{Li}$. To get the ${ }^{9} \mathrm{Li}$ energy equal to the experimental one, we have scaled the GPT potential by a factor of 0.37 . The spectroscopic factor corresponding to such a reduced interaction is shown in Fig. 2(b) by squares. Again, 

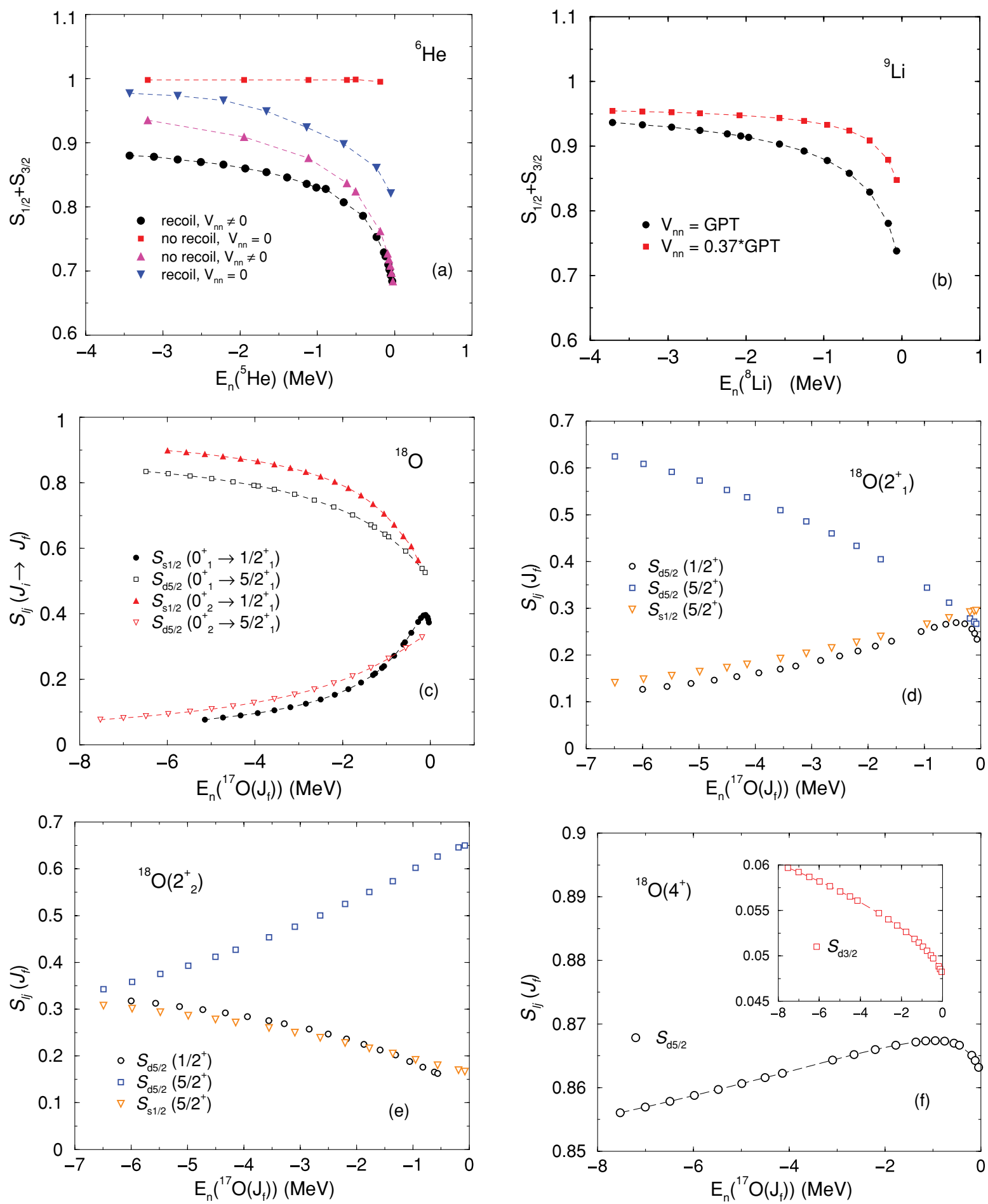

FIG. 2. (Color online) Spectroscopic factors $S_{l j}\left(J_{f}^{\pi}\right)$ of the core $+n+n$ nuclei as functions of bound-state energies $E_{n}$ in their core $+n$ subsystems with the spin-parity $J_{f}^{\pi}$ (see text for more details).

the threshold decrease in spectroscopic factor is clearly seen. As expected, the threshold effect is more pronounced when the $N N$ potential is stronger.

$$
\text { C. }{ }^{18} \mathrm{O}
$$

Unlike in two previous cases where only the $\left(l_{x}=\right.$ $\left.1, l_{y}=1\right)$ component gives significant contribution to the total wave function, there are two major components in ${ }^{18} \mathrm{O}\left(0^{+}\right),\left(l_{x}=0, l_{y}=0\right)$ and $\left(l_{x}=2, l_{y}=2\right)$. The splitting between them is governed by the coupled-channels problem (4). Because $\Psi_{3}$ is normalized to unity, any changes in spectroscopic factors of these components with the core$N$ binding energy are related. In particular, for the $n-{ }^{16} \mathrm{O}$ potential that reproduces the experimental energy of ${ }^{17} \mathrm{O}$, 


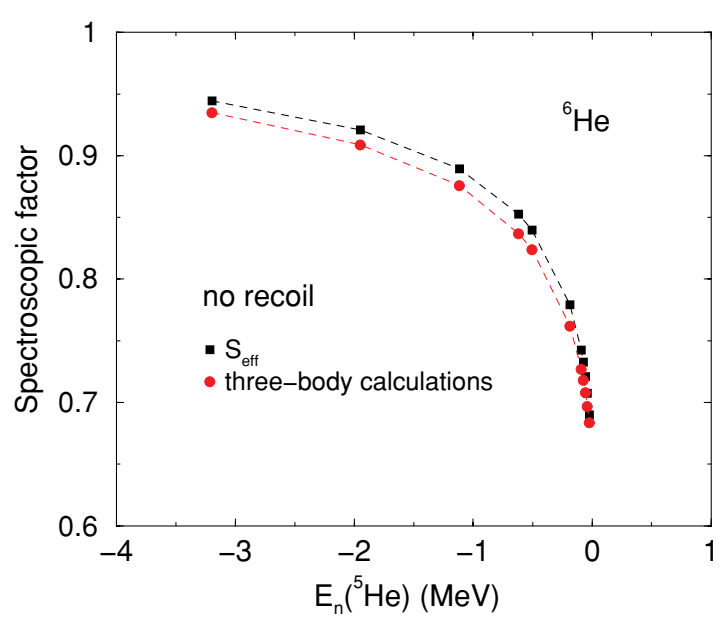

FIG. 3. (Color online) Spectroscopic factor from three-body calculations and its approximation $S_{\text {eff }}$ for infinitely large ${ }^{4} \mathrm{He}$ core.

the $d_{5 / 2}$ orbital is lower than $s_{1 / 2}$. Two neutrons in ${ }^{18} \mathrm{O}\left(0_{1}^{+}\right)$ prefer to occupy this orbital so that the spectroscopic factor $S_{d_{\frac{5}{2}}}\left(\frac{5}{2}^{+}\right)$, corresponding to the $\left[{ }^{17} \mathrm{O}\left(\frac{5}{2}^{+}\right) \otimes d_{\frac{5}{2}}\right]$ configuration is larger than $S_{s_{1}}\left(\frac{1}{2}^{+}\right)$corresponding to $\left[{ }^{17} \mathrm{O}\left(\frac{1}{2}^{+}\right) \otimes s_{\frac{1}{2}}\right]$. With decrease of the $n-{ }^{16} \mathrm{O}$ potential, the gap between the $d_{5 / 2}$ and $s_{1 / 2}$ decreases and inversion of the ${ }^{17} \mathrm{O}\left(\frac{5}{2}^{+}\right)$and ${ }^{17} \mathrm{O}\left(\frac{1}{2}^{+}\right)$levels occurs at $\left|E\left({ }^{17} \mathrm{O}\right)\right|<1.5 \mathrm{MeV}$. At this point, the $d_{5 / 2}$ orbital becomes no longer beneficial and the two nucleons occupy the $s_{1 / 2}$ orbital with larger probability. As the result, the spectroscopic factor $S_{S_{\frac{1}{2}}}\left(\frac{1}{2}^{+}\right)$increases with decrease of binding in ${ }^{17} \mathrm{O}$ causing $S_{d_{\frac{5}{2}}}\left(\frac{5^{+}}{2}\right)$ to decrease [see circles in Fig. 2(c)]. However, this tendency lasts only until very small values of $E\left({ }^{17} \mathrm{O}\right)$ where the near-threshold mismatch between the ${ }^{17} \mathrm{O}$ and the effective neutron wave functions begin to dominate, thus making $S_{s_{\frac{1}{2}}}\left(\frac{1}{2}^{+}\right)$to decrease again. As for the first excited state ${ }^{18} \mathrm{O}\left(0_{2}^{+}\right)$, the splitting of spectroscopic strength between $S_{S_{\frac{1}{2}}}\left(\frac{1}{2}^{+}\right)$and $S_{d_{\frac{5}{2}}}\left(\frac{5}{2}^{+}\right)$is different. This split, as well as the energy dependence, are influences by the probability for two neutrons to occupy the less favorable orbital. The dominant spectroscopic factor $S_{S_{\frac{1}{2}}}\left(\frac{1}{2}^{+}\right)$decreases all the time toward the threshold (triangles up), whereas the small spectroscopic factor $S_{d_{\frac{5}{2}}}\left(\frac{5}{2}^{+}\right)$increases (triangles down). The coupled-channels effects in the latter are stronger than the near-threshold mismatch between effective single-particle wave function and the two-body ${ }^{17} \mathrm{O}$ wave function so that no decrease is seen at $E\left({ }^{17} \mathrm{O}\right) \rightarrow 0$.

Similar situation occurs for excited $2^{+}$states [see Figs. 2(d) and 2(e)]. The coupling between the $\left[{ }^{17} \mathrm{O}\left(\frac{5}{2}^{+}\right) \otimes s_{\frac{1}{2}}\right]$, $\left[{ }^{17} \mathrm{O}\left(\frac{5}{2}^{+}\right) \otimes d_{\frac{5}{2}}\right]$, and $\left[{ }^{17} \mathrm{O}\left(\frac{1}{2}^{+}\right) \otimes d_{\frac{5}{2}}\right]$ configurations is very sensitive to the strengths of the "mean-field" $n-{ }^{16} \mathrm{O}$ potential. The interplay between the coupled-channels and threshold effects is clearly seen only in $S_{d_{\frac{5}{2}}}\left(\frac{1}{2}{ }^{+}\right)$ [open circles in Fig. 2(d)] when the residual nucleus ${ }^{17} \mathrm{O}$ has an $s$-wave halo neutron. In all other cases, it is difficult to separate these two effects. As for the ${ }^{18} \mathrm{O}\left(4^{+}\right)$ state, the $l=4$ coupling to the final ${ }^{17} \mathrm{O}\left(\frac{1}{2}^{+}\right)$state is negligible and, consequently, the spectroscopic factor $S_{d_{5}}\left(\frac{5}{2}^{+}\right)$ changes only within $2 \%$ over a large interval of the ${ }^{17} \mathrm{O}$ energies. A near-threshold decrease is clearly seen in Fig. 2(f) for this spectroscopic factor.

\section{Coulomb interaction}

It has been suggested in Ref. [6] that threshold anomaly in spectroscopic factors is also expected in proton-rich nuclei, although it should be weaker than in neutron-rich systems. However, no calculations confirming this suggestion have been presented. We note that the Coulomb barrier prevents the $p$-core wave function $\psi_{2}(r)$ to stretch too much toward the classically forbidden region at very small binding energies so that the mismatch between $\psi_{2}(r)$ and the effective singleproton wave function $\varphi_{\text {eff }}(r)$ should be much smaller. Therefore, the threshold effects should be indeed less pronounced. We demonstrate this in Fig. 4 for two selected cases, for ${ }^{6} \mathrm{Be}$, where all the spectroscopic strength is concentrated in the $p$ wave, and for the spectroscopic factor $S_{s_{1}}\left(\frac{1}{2}^{+}\right)$ in ${ }^{18} \mathrm{Ne}\left(0_{1}^{+}\right)$, the mirror analog of which experiences strong interference between the $s_{1 / 2}+d_{5 / 2}$ channels coupling and the near-threshold mismatch. The proton spectroscopic factors are shown as functions of the ${ }^{5} \mathrm{Li}$ and the ${ }^{17} \mathrm{~F}\left(\frac{1}{2}^{+}\right)$binding energies, respectively. They are compared with neutron spectroscopic factors that are plotted as functions of neutron separation energies. The near-threshold decrease is clearly seen in ${ }^{6} \mathrm{Be}$, although to a smaller extent than in ${ }^{6} \mathrm{He}$. However, the near-threshold decrease in ${ }^{18} \mathrm{Ne}\left(0_{1}^{+}\right)$disappears because the increase due to coupling between the $\left[{ }^{17} \mathrm{~F}\left(\frac{5}{2}^{+}\right) \otimes d_{5}\right]$ and $\left[{ }^{17} \mathrm{~F}\left(\frac{1}{2}^{+}\right) \otimes s_{\frac{1}{2}}\right]$ channels dominates. In fact, the ${ }^{16} \mathrm{O}-p$ interaction that weakly binds ${ }^{17} \mathrm{~F}\left(\frac{1}{2}^{+}\right)$, binds the the $d_{5 / 2}$ orbital stronger so that two protons in ${ }^{18} \mathrm{Ne}\left(0_{1}^{+}\right)$prefer to occupy it rather than the $s_{1 / 2}$ orbital. Therefore, the nearthreshold proton spectroscopic factor $S_{S_{\frac{1}{2}}}\left(\frac{1}{2}{ }^{+}\right)$is significantly

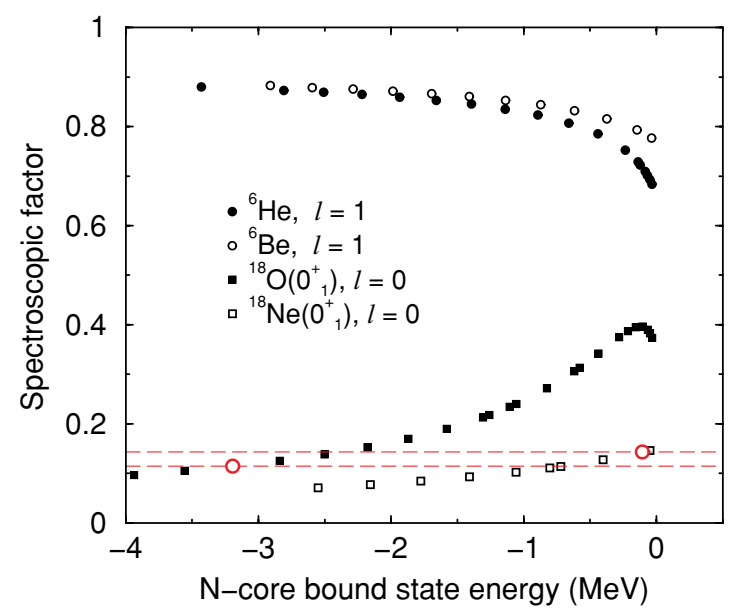

FIG. 4. (Color online) Neutron and proton spectroscopic factors for ${ }^{6} \mathrm{He},{ }^{6} \mathrm{Be},{ }^{18} \mathrm{O}\left(0_{1}^{+}\right)$, and ${ }^{18} \mathrm{Ne}\left(0_{1}^{+}\right)$for different bound-state energies of ${ }^{5} \mathrm{He},{ }^{5} \mathrm{Li},{ }^{17} \mathrm{O}\left(\frac{1}{2}^{+}\right)$, and ${ }^{17} \mathrm{~F}\left(\frac{1}{2}^{+}\right)$. Spectroscopic factors, corresponding to experimental energies of ${ }^{17} \mathrm{~F}\left(\frac{1}{2}^{+}\right)$and ${ }^{17} \mathrm{O}\left(\frac{1}{2}^{+}\right)$, are shown by large open circles marked by dashed lines. 
smaller than the neutron one corresponding to the same binding energy but to different ${ }^{16} \mathrm{O}-n$ interaction. The spectroscopic factors for mirror states ${ }^{18} \mathrm{Ne}\left(0_{1}^{+}\right)$and ${ }^{18} \mathrm{O}\left(0_{1}^{+}\right)$calculated at the same ${ }^{16} \mathrm{O}-n$ interaction, that gives the binding energies ${ }^{17} \mathrm{~F}\left(\frac{1}{2}^{+}\right)$and ${ }^{17} \mathrm{O}\left(\frac{1}{2}^{+}\right)$close to the experimential values, are shown in Fig. 4 by large circles marked by dashed lines. One can clearly see that these spectroscopic factors are not equal.

\section{ENERGY BEHAVIOR OF THE $\mathcal{R} / \mathcal{R}_{0}$ AND $S_{p} / S_{n}$ RATIOS}

We have calculated the ANCs $C_{p}$ and $C_{n}$ for the mirror overlaps $\langle$ core $+p|$ core $+p+p\rangle$ and $\langle$ core $+n|$ core $+n+$ $n\rangle$, corresponding to the same core- $N$ interaction, by using Eq. (7). With the chosen core- $N$ interactions the core $+p$ systems of interest remain bound. The $C_{p}$ and $C_{n}$ have the same quantum numbers $l j$ which are omitted below for simplicity. We compared the squared ratio of these ANCS, $\mathcal{R} \equiv\left(C_{p} / C_{n}\right)^{2}$, to the analytical estimate $\mathcal{R}_{0}$ given by Eq. (1). The ratios $\mathcal{R} / \mathcal{R}_{0}$ are represented in Fig. 5 by squares as the functions of the bound state energy of the core $+p$ system. These ratios are compared to the ratios $S_{p} / S_{n}$, where $S_{p}\left(S_{n}\right)$ is the proton (neutron) spectroscopic factor, in the same figure. The aim of such a comparison is to check whether a modified approximation,

$$
\mathcal{R} \approx \frac{S_{p}}{S_{n}} \mathcal{R}_{0}
$$

is sufficiently accurate. Approximate equality $\mathcal{R} / \mathcal{R}_{0} \approx S_{p} / S_{n}$ within 1-3\% means that effective local nuclear potential between the last proton and the $p+$ core system is the same as the effective potential between the mirror neutron and the $n+$ core system $[13,14]$.

\section{A. ${ }^{6} \mathrm{He}-{ }^{6} \mathrm{Be}$}

For this mirror pair, the ratio $\mathcal{R} / \mathcal{R}_{0}$, shown in Fig. 5(a), is very close to unity for large ${ }^{5} \mathrm{Li}$ binding energies but decreases at $E\left({ }^{5} \mathrm{Li}\right) \rightarrow 0$. Threshold effects reduce $\mathcal{R}$ against $\mathcal{R}_{0}$ by about $7 \%$. However, the ratio $S_{p} / S_{n}$ follows $\mathcal{R} / \mathcal{R}_{0}$ so that the modified approximation given by Eq. (10) holds within 1-2\%. The ratio $S_{p} / S_{n}$ can be approximated by the ratio of effective spectroscopic factors $S_{\text {eff }}\left({ }^{6} \mathrm{Be}\right)$ and $S_{\text {eff }}\left({ }^{6} \mathrm{He}\right)$ with accuracy of $1 \%$, which is shown by crosses in Fig. 5(a).

\section{B. ${ }^{9} \mathrm{Li}-{ }^{9} \mathrm{C}$}

The energy behavior of $\mathcal{R} / \mathcal{R}_{0}$ and $S_{p} / S_{n}$, calculated with GPT, is very similar to that obtained for the ${ }^{6} \mathrm{He}-{ }^{6} \mathrm{Be}$ case [see Fig. 5(b)], however, the threshold decrease of these quantities is weaker. Equation (10) works very well for all the energies too. With reduced GPT potential, the mismatch between effective wave function $\varphi_{\mathrm{eff}}(r)$ in ${ }^{9} \mathrm{Li}$ and the two-body ${ }^{8} \mathrm{Li}$ wave function is smaller, and, consequently, the threshold effects in $S_{p} / S_{n}$ are reduced and the symmetry breaking between $S_{p}$ and $S_{n}$ is less important. However, in ${ }^{9} \mathrm{C}$, reduced strength of the $N N$ potential makes the contributions from the Coulomb $p p$ force more important. This influences the
${ }^{9} \mathrm{C}$ ANC and leads to stronger deviations between $\mathcal{R}$ and $\mathcal{R}_{0}$. However, this deviation does not exceed $7 \%$. This means that the ${ }^{9} \mathrm{C}$ ANC obtained in Ref. [4] using the ${ }^{9} \mathrm{Li}$ ANC can be reduced due to the threshold effects by no more than $7 \%$.

\section{C. ${ }^{18} \mathrm{O}-{ }^{18} \mathrm{Ne}$}

As in two previous cases, the ratios $\mathcal{R} / \mathcal{R}_{0}$ and $S_{p} / S_{n}$ are, in general, close to unity when the binding energy of ${ }^{17} \mathrm{~F}$ is large and they deviate from unity toward the proton decay threshold in ${ }^{17} \mathrm{~F}$ [see Figs. 4(c)-4(g)]. However, due to different coupled-channels effects in the $l=0$ and $l=2$ configurations, the energy behavior of $\mathcal{R} / \mathcal{R}_{0}$ and $S_{p} / S_{n}$ becomes more complicated. In $0_{1,2}^{+}$states, the difference between $S_{p}$ and $S_{n}$ in large components does not exceed $5 \%$; however, it can reach up to $25 \%$ in the components with small spectroscopic factors. The ratio $\mathcal{R}$ deviates from $\mathcal{R}_{0}$ by $10 \%$ and $45 \%$ for the ground and the first excited $0^{+}$ states, respectively. The deviation between $\mathcal{R} / \mathcal{R}_{0}$ and $S_{p} / S_{n}$ ranges from $5-7 \%$ in large components to $15-20 \%$ in small components. This means that the $p-{ }^{17} \mathrm{~F}$ and the $n-{ }^{17} \mathrm{O}$ effective potential wells are different, especially in small components. In $2_{1,2}^{+}$states, the largest spectroscopic factor, $S_{d_{\frac{5}{2}}}\left(\frac{5}{2}^{+}\right)$, is only about factor of 2 larger than the other two, $S_{d_{\frac{5}{2}}}\left(\frac{1}{2}^{+}\right)$and $S_{S_{\frac{1}{2}}}\left(\frac{5}{2}^{+}\right)$, and the difference between mirror spêctroscopic factors ranges from 10 to $18 \%$. The $\mathcal{R} / \mathcal{R}_{0}$ correlates well with $S_{p} / S_{n}$ for the $\left[\frac{5}{2}^{+} \otimes d_{\frac{5}{2}}\right]$ configuration, whereas deviations between $\mathcal{R} / \mathcal{R}_{0}$ and $S_{p} / S_{n}$ can reach up to $12 \%$ for two other configurations. In the $4^{+}$state, $\mathcal{R} / \mathcal{R}_{0}$ and $S_{p} / S_{n}$ are close to unity for the main configuration $\left[\frac{5}{2}^{+} \otimes d_{\frac{5}{2}}\right]$ but in small configuration $\left[\frac{5}{2}^{+} \otimes d_{\frac{3}{2}}\right]$ the symmetry breaking in spectroscopic factors is about $15 \%$. However, the approximation $\mathcal{R} / \mathcal{R}_{0} \approx S_{p} / S_{n}$ works reasonably well in this case.

\section{DISCUSSION AND CONCLUSIONS}

The present study of the spectroscopic factors in threebody systems core $+N+N$ has revealed that at small binding energies of the two-body subsystems core $+N$, where the correlations between the two nucleons outside the core become very important, these spectroscopic factors exhibit a specific behavior first discovered by the Gamow shell-model calculations in Ref. [6]. Such a behavior can be the most clearly seen when the Coulomb interactions and the coupling between the channels with different orbital momentum are absent. In these cases, for example in ${ }^{6} \mathrm{He}$ and ${ }^{9} \mathrm{Li}$, the spectroscopic factors strongly decrease toward the neutron decay threshold.

The origin of such a decrease is the strong $N N$ interaction that binds the three-body system with an energy significantly exceeding twice the energy of the core $+N$ subsystem. As the result, the three-body wave function $\Psi_{3}$ is concentrated in a spatially confined area, whereas the two-body wave function $\psi_{2}$ stretches out of this area into the classically forbidden region. The internal norm of $\psi_{2}$ dramatically decreases at small 

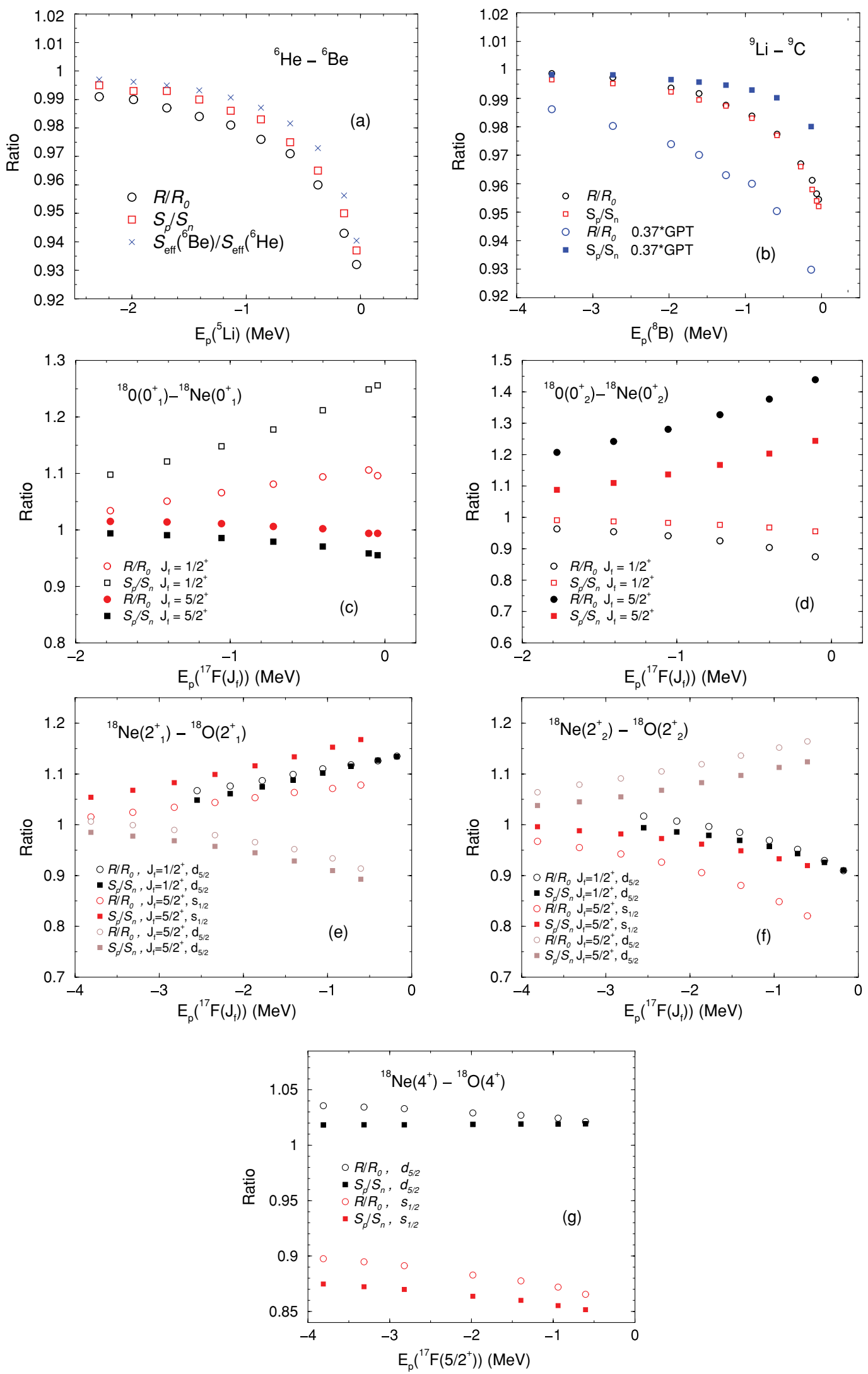

FIG. 5. (Color online) Ratios $\mathcal{R} / \mathcal{R}_{0}$ (squares) and $S_{p} / S_{n}$ (circles) for different bound-state energies $E_{p}$ in the core $+p$ subsystem, calculated for various mirror three-body nuclei. For the $0^{+}$and $2^{+}$states in ${ }^{18} \mathrm{Ne}^{18} \mathrm{O}, E_{p}$ corresponds either to ${ }^{17} \mathrm{~F}\left(\frac{5}{2}^{+}\right)$, if $J_{f}=\frac{5}{2}^{+}$, or to ${ }^{17} \mathrm{~F}\left(\frac{1}{2}^{+}\right)$, if $J_{f}=\frac{1}{2}^{+}$. 
core $+N$ binding energies and so does the overlap between $\Psi_{3}$ and $\psi_{2}$, the norm of which is the spectroscopic factor. This effect is the best seen for the $s$-wave halo core $+n$ systems where $S_{s_{1}} \rightarrow 0$ at $E_{\text {core }-n} \rightarrow 0$. If the centrifugal or/and the Coulomb barriers are present in the $N$-core interaction, then internal norm of $\psi_{2}$ decreases with binding energy more slowly so that the near-threshold spectroscopic factor's decrease is less pronounced. The energy dependence of these spectroscopic factors can be successfully modeled by overlapping $\psi_{2}$ with a Hartree-Fock type wave function $\Psi_{\mathrm{HF}}$ that can be considered as an approximation to $\Psi_{3}$. $\Psi_{\mathrm{HF}}$ is an antisymmetrized product of two effective single-particle wave functions $\varphi_{\text {eff }}$ that belong to a mean-field Hamiltonian that differs from the original $n$-core Hamiltonian. This effective Hamiltonian accounts for the increase of binding due to the strong $N N$ correlations and provides correct asymptotic forms for the $\langle$ core $+N|$ core + $N+N\rangle$ overlap. The resulting effective spectroscopic factor is a measure of the mismatch between $\varphi_{\text {eff }}$ and $\psi_{2}$. ${ }^{1}$ It may need a further small energy-independent reduction $\left(10 \%\right.$ for the ${ }^{6} \mathrm{He}$ case) but with increasing mass of the core this reduction is expected to become insignificant. It is interesting to note here that the core recoil is another factor that significantly influences the energy behavior of spectroscopic factors. It can cause the near-threshold decrease even in the absence of any $N N$ interaction.

The energy behavior of spectroscopic factors becomes more complicated when the spectroscopic strength is split between the channels with different orbital momentum. The coupling between channels may cause an increase of spectroscopic factors toward the threshold, in which cases the near-threshold decrease can be visible only for $s$-wave core $+n$ systems. For other cases, the near-threshold decrease either does not happen or cannot be distinguished from coupled-channels effects.

The simultaneous consideration of mirror systems has confirmed the possibility of symmetry breaking in mirror spectroscopic factors, which becomes more important at low $p$-core binding energies. It arises because at low $p$-core binding energies the proton spectroscopic factors are influenced by the threshold effects, whereas in the mirror system the $n$-core energy is always larger, so that the mirror neutron spectroscopic factor is not influenced by the near-threshold effects in the same extent. For realistic $N$-core energies, the actual mirror symmetry breaking can be as small as $2-5 \%$ in large components but it can reach unusually large values, up to $25 \%$, which occurs in small component $\left[\frac{1}{2}^{+} \otimes s_{\frac{1}{2}}\right]$ of the $0_{1}^{+}$states in ${ }^{18} \mathrm{O}_{-}{ }^{18} \mathrm{Ne}$. This is a large effect that can be experimentally measurable, for example, using knockout reaction from ${ }^{18} \mathrm{O}$ and ${ }^{18} \mathrm{Ne}$.

The symmetry breaking in mirror spectroscopic factors leads to deviation of the ratio of mirror ANCs from the prediction of the simple analytical formula (1). When coupling

\footnotetext{
${ }^{1}$ The idea of geometrical mismatch between single-particle wave functions in initial and final states studied via knockout reactions were first used by A. Navin et al. in Phys. Rev. Lett. 85, 266 (2000) when deriving the spectroscopic factor of ${ }^{12} \mathrm{Be}$. The mismatch factor is also discussed by P. G. Hansen and J. A. Tostevin in Annu. Rev. Nucl. Part. Sci. 53, 219 (2003).
}

between channels with different orbital momentum is absent, the $\mathcal{R} / \mathcal{R}_{0}$ follows $S_{p} / S_{n}$ so that the modified formula (10) can be used to predict proton ANCs and the corresponding stellar $(p, \gamma)$ cross sections from the measured mirror neutron ANC. For this purpose, the ratio $S_{p} / S_{n}$ can be estimated in the three-body model, or modeled by a ratio of effective spectroscopic factors to take the mismatch between $\psi_{2}$ and $\psi_{\text {eff }}$ into account.

The deviations between $\mathcal{R} / \mathcal{R}_{0}$ and $S_{p} / S_{n}$ can become significant if several channels with different orbital momentum are important in the three-body wave function $\Psi_{3}$. This can create problems for predictions of the $(p, \gamma)$ cross sections from mirror neutron ANCs. Such problems are particularly expected when only one channel contributes to the $(p, \gamma)$ cross sections. However, if several channels equally contribute to the $(p, \gamma)$ cross section and if $\mathcal{R} / \mathcal{R}_{0}$ averaged over these channels is approximately equal to 1 , then inaccuracies of the $(p, \gamma)$ predictions in individual channels can cancel out. In the present study, the strongest deviation between $\mathcal{R} / \mathcal{R}_{0}$ and $S_{p} / S_{n}$ is obtained for the small component $\left[\frac{5}{2}^{+} \otimes d_{\frac{5}{2}}\right]$ in the $0_{2}^{+}$state in ${ }^{18} \mathrm{O}_{-}{ }^{18} \mathrm{Ne}, \mathcal{R} / \mathcal{R}_{0}=1.45$ and $\mathcal{R} / \mathcal{R}_{0} \cdot S_{n} / S_{p}=$ 1.16. Fortunately, the $0_{2}^{+}$level is not populated in novae nucleosynthesis; however, the population of the $2_{1,2}^{+}$states in ${ }^{18} \mathrm{Ne}$ is important. For the $\left[\left(\frac{1}{2}^{+}\right) \otimes d_{\frac{5}{2}}\right]$ component of these states the modified formula (10) is accurate within the 3-5\%; however, predictions for the population of the $\left[\frac{5}{2}^{+} \otimes s_{\frac{1}{2}}\right]$ components can be inaccurate up to $10-12 \%$. For $4^{+}$state, the assumptions of mirror symmetry can be safely used.

Finally, all the conclusions of the present study are based on the restricted three-body core $+2 N$ model, which is, in general, incomplete for complex nuclei. Although the limits of applicability of three-body models are not completely understood, they are actively used in many physical applications. Based on the present study, we propose a new tool that may allow us to "measure" the applicability of the three-body models to realistic nuclei. We predict a new phenomenon that arises entirely due to dynamical three-body effects. This phenomenon, the symmetry breaking in mirror spectroscopic factors and ANCs, in some cases is sufficiently strong to be observable. In particular, the $45 \%$ difference between the ratio of mirror ANCs in ${ }^{18} \mathrm{O}\left(0_{2}^{+}\right)-{ }^{18} \mathrm{Ne}\left(0_{2}^{+}\right)$and the analytical prediction $\mathcal{R}_{0}$ can be searched for in the ${ }^{17} \mathrm{O}(d, p){ }^{18} \mathrm{O}\left(0_{2}^{+}\right)$and ${ }^{17} \mathrm{~F}(d, n){ }^{18} \mathrm{Ne}\left(0_{2}^{+}\right)$reactions. Experimental confirmation or rejection of this phenomenon will contribute to our knowlegde about proper choice of theoretical models relevant to realistic nuclear systems and, therefore, is a timely and extremely important task.

\section{ACKNOWLEDGMENTS}

N.K.T. thanks L. Grigorenko for useful discussions. This work was performed under the UK grants EP/C520521/1 and EP/E036627/1 and in the Lawrence Livermore National Laboratory under Department of Energy contract DE-AC5207NA27344. 
[1] H. M. Xu, C. A. Gagliardi, R. E. Tribble, A. M. Mukhamedzhanov, and N. K. Timofeyuk, Phys. Rev. Lett. 73, 2027 (1994).

[2] N. K. Timofeyuk, R. C. Johnson, and A. M. Mukhamedzhanov, Phys. Rev. Lett. 91, 232501 (2003).

[3] B. Guo, Z. H. Li, W. P. Liu, X. X. Bai, G. Lian, S. Q. Yan, B. X. Wang, S. Zeng, J. Su, and Y. Lu, Nucl. Phys. A761, 162 (2005).

[4] B. Guo, Z. H. Li, X. X. Bai, W. P. Liu, N. C. Shu, and Y. S. Chen, Phys. Rev. C 73, 048801 (2006).

[5] B. Guo, Z. H. Li, W. P. Liu, and X. X. Bai, J. Phys. G 34, 103 (2007).

[6] N. Michel, W. Nazarewicz, and M. Ploszajczak, Phys. Rev. C 75, 031301(R) (2007); Nucl. Phys. A794, 29 (2007).

[7] N. K. Timofeyuk, L. D. Blokhintsev, and J. A. Tostevin, Phys. Rev. C 68, 021601(R) (2003).
[8] N. K. Timofeyuk, I. J. Thompson, and J. A. Tostevin, J. Phys. Conf. Ser. 111, 012034 (2008).

[9] J. M. Bang, B. V. Danilin, V. D. Efros, J. S. Vaagen, M. V. Zhukov, I. J. Thompson, and the Russian-Nordic-British Theory (RNBT) Collaboration, Phys. Rep. 264, 27 (1996).

[10] I. J. Thompson, B. V. Danilin, V. D. Efros, J. S. Vaagen, J. M. Bang, and M. V. Zhukov, Phys. Rev. C 61, 024318 (2000).

[11] D. Gogny, P. Pires, and R. De Tourreil, Phys. Lett. B32, 591 (1970).

[12] L. V. Grigorenko, B. V. Danilin, V. D. Efros, N. B. Shul'gina, and M. V. Zhukov, Phys. Rev. C 60, 044312 (1999).

[13] N. K. Timofeyuk and P. Descouvemont, Phys. Rev. C 71, 064305 (2005).

[14] N. K. Timofeyuk, P. Descouvemont, and R. C. Johnson, Eur. Phys. J. A 27, Supplement 1, 269 (2006). 